\title{
JAMES MAPS AND $E_{n}$ RING SPACES \\ BY \\ F. R. COHEN, J. P. MAY AND L. R. TAYLOR
}

ABSTRACT. We parametrize by operad actions the multiplicative analysis of the total James map given by Caruso and ourselves. The target of the total James map

$$
j=\sum j_{q}: C\left(R^{n}, X\right) \rightarrow \prod_{q \geqslant 0} Q D_{q}\left(R^{n}, X\right)
$$

is an $E_{n}$ ring space and $j$ is a $\bigcup_{n}$-map, where $\bigcup_{n}$ is the little $n$-cubes operad. This implies that $j$ has an $n$-fold delooping with domain $\Sigma^{n} X$. It also implies an algorithm for the calculation of $j_{*}$ and thus of each $\left(j_{q}\right)_{*}$ on $\bmod p$ homology. When $n=\infty$ and $p=2$, this algorithm is the essential starting point for Kuhn's proof of the Whitehead conjecture.

In $[2, \S 4]$, we analyzed the behavior with respect to pairings of the James maps $j_{q}: C X \rightarrow Q D_{q}(\mathcal{C}, X)$ used in [4] to obtain the stable splittings of spaces $C X$. In particular, we proved that if the given coefficient system $\mathcal{C}$ has a sum, then the product of the targets is a ring space and the product of the $j_{q}$ 's is an exponential $H$-map. In fact, this is only a fragment of the full structure present in the most interesting examples.

In the first two sections, we give a parametrized elaboration of this analysis. We assume the given coefficient system $\mathcal{C}$ is a "module" over an operad $\mathcal{Q}$ and we deduce, essentially, that

$$
\left(j_{q}\right): C X \rightarrow \prod_{q \geqslant 0} Q D_{q}(\bigodot, X)
$$

is a G-map. Here $\Pi_{q \geq 0}$ denotes the weak infinite product (all but finitely many coordinates of each point are the basepoint). Technically, we shall replace the target by its equivalent $Q\left(\vee_{q \geqslant 0} D_{q}(\mathcal{C}, X)\right)$ and $\mathcal{G}$ by an equivalent, but larger, operad.

This analysis requires the introduction of general notions of additive and multiplicative actions of operads on coefficient systems, and these generalizations of the definitions of an operad and of an action of one operad on another give considerable added flexibility to the theory of iterated loop spaces developed in [9-13].

When $\mathcal{C}$ is $\mathcal{C}\left(R^{n}\right)$, our results imply that $\left(j_{q}\right)$ has an $n$-fold delooping

$$
\Sigma^{n} X \rightarrow B^{n}\left(\prod_{q \geqslant 1} Q D_{q}\left(R^{n}, X\right)\right) .
$$

Received by the editors January 20, 1983.

1980 Mathematics Subject Classification. Primary 55P35, 55P47, 55S12; Secondary 55Q25.

Key words and phrases. James maps, $E_{n}$ ring space, operad, coefficient system, homology operations. 
In the case $n=2$, this can be improved to a 2-fold delooping

$$
\Sigma^{2} X \rightarrow B^{2}\left(\prod_{q \geqslant 1} C\left(R^{2 q}, D_{q}\left(R^{2}, X\right)\right)\right),
$$

and $C\left(R^{2 q}, D_{q}\left(R^{2}, X\right)\right)$ may be replaced by $\Omega^{2 q} \Sigma^{2 q} D_{q}\left(R^{2}, X\right)$ if $X$ is path connected. Moreover, the sequence of maps $j_{q}: C\left(R^{n}, X\right) \rightarrow Q D_{q}\left(R^{n}, X\right)$ is entirely determined by the multiplicative structure of the " $E_{n}$ ring space" $\Pi_{q \geqslant 0} Q D_{q}\left(R^{n}, X\right)$. This fact implies an algorithm for the calculation of the maps $j_{q}$ on homology. We discuss these implications in \$3. Kuhn [6] has made extensive mod 2 calculations on the basis of the cited algorithm, and he has recently used these calculations to construct a homotopical resolution of the sphere spectrum [7], thus generalizing the Kahn-Priddy Theorem and proving a long-standing conjecture of G. W. Whitehead.

Ralph. Cohen has pointed out that the spectrum level analogs of the basic constructions $C X$ and $D_{q}(\mathcal{C}, X)$ can be used to obtain a totally noncombinatorial conceptual construction of the space level James maps and to prove their multiplicative properties. The main ideas are explained in his paper [5]. We originally planned to say more about this here but have decided to defer the discussion to [8], where the foundational work necessary to make it rigorous is carried out.

This is a sequel to [2], but only $\$ \S 2-4$ of that paper are essential preliminaries to this one.

1. Additive actions of operads on coefficient systems. We here relate the canonical James maps of separated coefficient systems to operad actions. As we shall see in a moment, the following is a natural generalization of the definition of an operad $[9$, p. 1].

DEFINITION 1.1. Let $\mathcal{G}$ be an operad and $\mathcal{C}$ a coeffcient system. Then $\mathcal{C}$ is said to be a $\mathcal{G}$-module if there are maps

$$
\gamma: \mathfrak{G}_{k} \times \mathfrak{e}_{j_{1}} \times \cdots \times \mathfrak{e}_{j_{k}} \rightarrow \mathfrak{e}_{j}, \quad j=j_{1}+\cdots+j_{k},
$$

which satisfy the following properties.

(a) If $g \in \mathcal{G}_{k}, h_{r} \in \mathcal{G}_{j_{r}}$ for $1 \leqslant r \leqslant k$, and $c_{s} \in \mathcal{C}_{i_{s}}$ for $1 \leqslant s \leqslant j$, then

$$
\gamma\left(\gamma\left(g ; h_{1}, \ldots, h_{k}\right) ; c_{1}, \ldots, c_{j}\right)=\gamma\left(g ; d_{1}, \ldots, d_{k}\right),
$$

where $d_{r}=\gamma\left(h_{r} ; c_{j_{1}+\cdots+j_{r-1}+1}, \ldots, c_{j_{1}+\cdots+j_{r}}\right)$ with $d_{r}=*$ if $j_{r}=0$.

(b) $\gamma(1 ; c)=c$, where $1 \in \mathcal{G}_{1}$ is the unit of $\mathcal{G}$.

(c) If $g \in \mathcal{G}_{k}, c_{r} \in \mathcal{C}_{j_{r}}$, and $\sigma \in \Sigma_{k}$, then

$$
\gamma\left(g \sigma ; c_{1}, \ldots, c_{k}\right)=\gamma\left(g ; c_{\sigma^{-1}(1)}, \ldots, c_{\sigma^{-1}(k)}\right) \sigma\left(j_{1}, \ldots, j_{k}\right),
$$

where $\sigma\left(j_{1}, \ldots, j_{k}\right) \in \Sigma_{j}$ is the permutation

$$
\mathbf{j} \cong \mathbf{j}_{1} \vee \cdots \vee \mathbf{j}_{k} \stackrel{\sigma}{\rightarrow} \mathbf{j}_{\sigma^{-1}(1)} \vee \cdots \vee \mathbf{j}_{\sigma^{-1}(k)} \cong \mathbf{j}
$$

the isomorphisms being block sum identifications.

(d) For each fixed $g \in \mathcal{G}_{k}$, the maps $\gamma(g)$ specify a $k$-fold sum on $\mathcal{C}$, that is, a natural transformation $\mathcal{C}^{k} \rightarrow \mathcal{C} \circ \vee$, where $\vee: \Lambda^{k} \rightarrow \Lambda$ is the $k$-fold wedge. 
Definition 1.2. A $\mathcal{G}$-module $\mathcal{C}$ is said to be separated if $\mathcal{C}$ is separated and if each sum $\gamma(g)$ is separated in the sense that the maps

$$
\gamma(g): \prod_{j_{1}+\cdots+j_{k}=j} \bar{e}_{j_{1}} \times \cdots \times \overline{\mathrm{e}}_{j_{k}} \rightarrow \overline{\mathrm{e}}_{j}
$$

induced by passage to orbit spaces are all injections; $\mathcal{C}$ is said to be weakly separated if each $\gamma(g)$ is weakly separated in the sense that these orbit maps induce a $k$-fold pairing on $\left\{\mathcal{C}\left(\overline{\mathcal{C}}_{j}\right)\right\}$; compare $[2,4.1]$.

EXAMPLE 1.3. When $\mathcal{C}=\mathcal{G}$, only the right unit formula $\gamma\left(g ; 1^{k}\right)=g$ needs to be added to make Definition 1.1 coincide with the definition of an operad. In particular, an operad $\mathcal{G}$ is a $\mathcal{G}$-module. The little cubes operad $\mathcal{C}_{n}$ is a separated $e_{n}$-module.

EXAMPLE 1.4. Let $\mathcal{G}=\mathcal{C}_{n}$ and let $\mathcal{e}=\mathcal{C}\left(J^{n} \times Y\right)$ for any space $Y$, where $J$ is the open unit interval. Then $\mathcal{C}$ is a separated $\mathcal{G}$-module with respect to the maps $\gamma$ specified as follows. For a little cube $f: J^{n} \rightarrow J^{n}$ and an element $b=\left\langle z_{1}, \ldots, z_{j}\right\rangle$ of $F\left(J^{n} \times Y, j\right)$ with $z_{s}=\left(x_{s}, y_{s}\right), x_{s} \in J^{n}$ and $y_{s} \in Y$, define

$$
f b=\left\langle\left(f x_{1}, y_{1}\right), \ldots,\left(f x_{j}, y_{j}\right)\right\rangle \text {. }
$$

Then define

$$
\gamma\left(g ; c_{1}, \ldots, c_{k}\right)=\left\langle g_{1} c_{1}, \ldots, g_{k} c_{k}\right\rangle \in F\left(J^{n} \times Y, j_{1}+\cdots+j_{k}\right)
$$

for $g=\left\langle g_{1}, \ldots, g_{k}\right\rangle \in \mathcal{C}_{n, k}$ and $c_{r} \in F\left(J^{n} \times Y, j_{r}\right), 1 \leqslant r \leqslant k$. These maps $\gamma$ are precisely analogous to the structural maps $\gamma$ of $\bigodot_{n}$, and centerpoint projection specifies morphisms $g_{n}: e_{n} \rightarrow \mathcal{C}\left(J^{n}\right)$ of $\bigcup_{n}$-modules.

Henceforth we write $R$ instead of $J$, by abuse.

EXAMPLE 1.5. If $\mathcal{C}$ is a $\mathcal{G}$-module and $\mathcal{C}^{\prime}$ is a $\mathcal{G}^{\prime}$-module, then $\mathcal{C} \times \mathcal{C}^{\prime}$ is a $\mathcal{G} \times \mathcal{G}^{\prime}$-module. If $\mathcal{C}$ or $\mathcal{C}^{\prime}$ is weakly separated, then so is $\mathcal{C} \times \mathcal{C}^{\prime}$. (The analogous assertion with "weakly separated" replaced by "separated" is false.)

Recall from [9, p. 5] that an action of an operad $\mathcal{G}$ on a space $X$ with basepoint 1 is a sequence of $\Sigma_{k}$-equivariant maps $\xi_{k}: \mathcal{G}_{k} \times X^{k} \rightarrow X$ such that $\xi_{0}(*, *)=1, \xi_{1}(1, x)$ $=x$, and

$$
\xi_{j}\left(\gamma\left(g ; h_{1}, \ldots, h_{k}\right) ; x_{1}, \ldots, x_{j}\right)=\xi_{k}\left(g ; \xi_{j_{1}}\left(h_{1}, y_{1}\right), \ldots, \xi_{j_{k}}\left(h_{k}, y_{k}\right)\right)
$$

for $g \in \mathcal{G}_{k}, h_{r} \in \mathcal{G}_{j_{r}}$ and $x_{s} \in X$, where $j=j_{1}+\cdots+j_{k}$ and $y_{r}$ is the $r$ th block of $j_{r}$

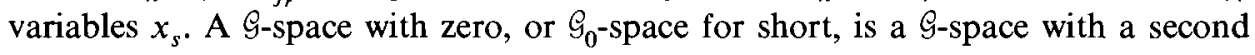
basepoint 0 such that $\xi_{k}\left(g ; x_{1}, \ldots, x_{k}\right)=0$ if any $x_{r}=0$. Thus $\xi_{k}$ induces a based map $D_{r}(\mathcal{G}, X) \rightarrow X$, where the smash powers of $X$ are taken with respect to the basepoint 0 .

We need a number of structures implied by Definitions 1.1 and 1.2. First, we have

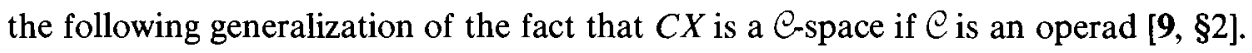

LEMma 1.6. Let $\mathcal{C}$ be a $\mathcal{G}$-module. For based spaces $X, C X$ is functorially a $\mathcal{G}$-space.

Proof. For $g \in \mathcal{G}_{k}, c_{r} \in \mathcal{C}_{j_{r}}$, and $y_{r} \in X^{j_{r}}$, define

$$
\xi_{k}\left(g ;\left[c_{1} ; y_{1}\right], \ldots,\left[c_{k} ; y_{k}\right]\right)=\left[\gamma\left(g ; c_{1}, \ldots, c_{k}\right) ; y_{1}, \ldots, y_{k}\right] \text {. }
$$

The requisite verifications are trivial. 
Lemma 1.7. Let $\mathcal{C}$ be a 9 -module. For a based space $X$, define

$$
D(\Theta, X)=\underset{q \geqslant 0}{\vee} D_{q}(e, X)
$$

Then $D(\mathcal{E}, X)$ is functorially a $\mathfrak{G}_{0}$-space.

Proof. $D_{0}(e, X)=S^{0}$, the wedge uses the basepoint 0 , and the point 1 is to be the unit for the $G$-action. The maps in the previous proof pass to quotients to give $\Sigma_{k}$-equivariant maps

$$
\mathcal{G}_{k}^{+} \wedge D_{j_{1}}(\circlearrowright, X) \wedge \cdots \wedge D_{j_{k}}(\circlearrowright, X) \rightarrow D_{j}(e, X),
$$

$j=j_{1}+\cdots+j_{k}$, and these provide the required action.

LEMMA 1.8. Let $\mathrm{C}$ be a weakly separated $\mathrm{G}$-module and define

$$
\overline{\mathrm{e}}=\coprod_{q \geqslant 0} \overline{\mathrm{e}}_{q} \text {, where } \overline{\mathrm{e}}_{q}=\mathrm{e}_{q} / \Sigma_{q} \text { with } \overline{\mathrm{e}}_{0}=\{*\} \text {. }
$$

For $\mathcal{G}_{0}$-spaces $X, C(\bar{\varrho}, X)$ is functorially a $\mathfrak{G}_{0}$-space.

Proof. $C(\bar{e}, X)$ is defined using the basepoint $0 \in X$, and the unit of $C(\overline{\mathcal{C}}, X)$ is the element $[\langle *\rangle ; 1]$. For $g \in \mathcal{G}_{k}$, the action of $g$ is the "composite"

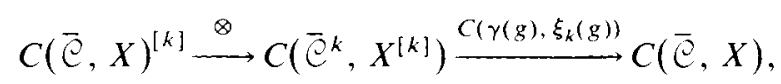

where $\otimes$ is given by $[2,1.9]$. The weak separation property is just enough to ensure that the second map is defined on the image of the first; it is only defined on the entire middle space when $\mathcal{C}$ is a separated $\mathcal{G}$-module. Continuity in $g$ is clear, and the requisite associativity, unity, and equivariance formulas are inherited from those of $\gamma$ and the $\xi_{k}$.

When $X=\vee_{q \geqslant 0} X_{q}$, the external sum $\oplus: \vee_{q \geqslant 0} C\left(\bar{\bigodot}_{q}, X_{q}\right) \rightarrow C(\bar{\varrho}, X)$ of $[2,2.5]$ is an inclusion. Its image consists of those points $\left[\left\langle c_{1}, \ldots, c_{k}\right\rangle ; x_{1}, \ldots, x_{k}\right]$ which are diagonally paired in the sense that $c_{r} \in \overline{\mathcal{C}}_{q}$ if and only if $x_{r} \in X_{q}$, for the same $q$. Provided that $\xi_{k}(g)$ carries $X_{j_{k}} \wedge \cdots \wedge X_{j_{k}}$ to $X_{j}, j=j_{1}+\cdots+j_{k}$, this subspace is closed under the $\mathcal{G}$-action. On the domain of $\oplus, \mathcal{G}_{k}$ may then be viewed as a parameter space indexing $k$-fold multiplications $\square$ of the sort introduced in [2, \$2]. We have chosen to work in the larger space for conceptual clarity and for technical convenience in the next section.

When $C$ is a separated coefficient system, we agree to regard the following composite as the canonical total James map $j$ :

$$
C X \stackrel{\left(j_{q}\right)}{\rightarrow} \prod_{q \geqslant 0} C\left(\overline{\mathfrak{e}}_{q}, D_{q}(\mathcal{e}, X)\right) \stackrel{\oplus}{\rightarrow} C(\overline{\mathfrak{e}}, D(\mathcal{e}, X))
$$

compare [2, \$4]. When $\circlearrowright=\bigodot\left(J^{n}\right), j$ is induced by sending a finite subset of $J^{n}$ to its power set (see $[2, \S 6]$ ) and is therefore exponential. The following basic observation is then just a parametrization of this fact by little cubes.

Proposirion 1.9. Let $巳$ be a weakly separated G-module. Then $j: C X \rightarrow$ $C(\bar{\circlearrowright}, D(\mathcal{E}, X))$ is a map of $\mathcal{G}$-spaces. 
Proof. It suffices to verify that the following diagram commutes for $g \in \mathcal{G}_{k}$ :

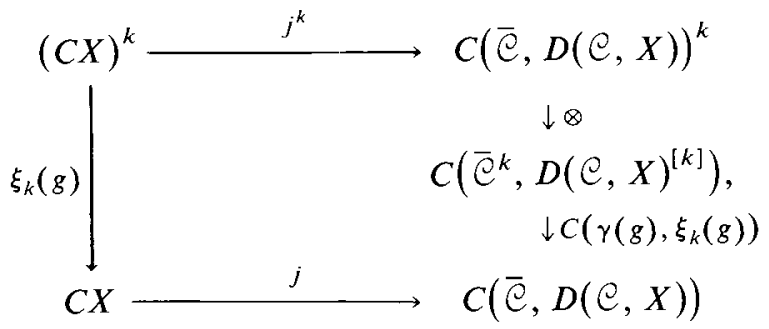

The results of $[2, \S \S 2-4]$ admit evident generalizations to $k$-fold pairings, and our diagram is just that of $[2,3.4]$ as obtained from the $k$-fold weakly separated sum $\gamma(g): e^{k} \rightarrow e$. Thus it commutes by $[2,4.2]$.

While we shall generally use this result in the form given, we can of course restrict the target to the domain of $\oplus$. This is advantageous in the following example.

EXAMPLE 1.10. Consider $\mathcal{C}\left(R^{n}\right) \subset \mathscr{P}\left(R^{n}\right)$, where $\mathscr{P}\left(R^{n}\right)_{q}=R^{n q}$. While $\mathscr{P}\left(R^{n}\right)$ is of course not separated, it does satisfy the injectivity condition required of separated $e_{n}$-modules in Definition 1.2. Therefore Lemma 1.8 applies to it and we have maps of $e_{n}$-spaces:

$$
C\left(R^{n}, X\right) \stackrel{\left(J_{q}\right)}{\rightarrow} \prod_{q \geqslant 0} C\left(B\left(R^{n}, q\right), D_{q}\left(R^{n}, X\right)\right) \rightarrow \prod_{q \geqslant 0} C\left(R^{n q} / \Sigma_{q}, D_{q}\left(R^{n}, X\right)\right) .
$$

Take $n=2$. Here $R^{2 q} / \Sigma_{q} \cong \mathrm{C}^{q} / \Sigma_{q} \cong \mathrm{C}^{q} \cong R^{2 q}$ by the fundamental theorem of algebra. This implies a "delooping"

$$
\Sigma^{2} X \rightarrow B^{2}\left(\{1\} \times \prod_{q \geqslant 1} C\left(R^{2 q}, D_{q}\left(R^{2}, X\right)\right)\right) .
$$

(See the discussion in §3.)

2. Multiplicative actions of operads on coefficient systems. We want to transport Proposition 1.9 along the chain of maps connecting its target to $Q D(\mathcal{C}, X)$. For this purpose, we reinterpret the action of $\mathcal{G}$ on the target in terms of the following definition.

Definition 2.1. Let $\mathcal{G}$ be an operad and $\mathcal{C}$ a coefficient system. Then $\mathcal{G}$ is said to act multiplicatively on $e$ if there is a unit element $1 \in \Theta_{1}$ and there are maps $\lambda: \mathfrak{G}_{k} \times \bigodot_{j_{1}} \times \cdots \times \bigodot_{j_{k}} \rightarrow \mathcal{C}_{j}, j=j_{1} \cdots j_{k}$, which satisfy the following properties.

(a) If $g \in \mathcal{G}_{k}, h_{r} \in \mathcal{G}_{j_{r}}$ for $1 \leqslant r \leqslant k$, and $c_{s} \in \mathcal{C}_{l_{s}}$ for $1 \leqslant s \leqslant j_{1}+\cdots+j_{k}$, then

$$
\lambda\left(\gamma\left(g ; h_{1}, \ldots, h_{k}\right), c_{1}, \ldots, c_{j_{1}+\cdots+j_{k}}\right)=\lambda\left(g ; d_{1}, \ldots, d_{k}\right),
$$

where $d_{r}=\lambda\left(h_{r} ; c_{j_{1}+\cdots+j_{r-1}+1}, \ldots, c_{j_{1}+\cdots+j_{r}}\right)$ with $d_{r}=1$ if $j_{r}=0$.

(b) $\lambda(1, c)=c$ for $c \in \mathcal{C}_{j}$ and $\lambda\left(g ; 1^{k}\right)=1$ for $g \in \mathcal{G}_{k}$.

(c) If $g \in \mathcal{G}_{k}, c_{r} \in \mathcal{G}_{j_{r}}$ and $\sigma \in \Sigma_{k}$, then

$$
\lambda\left(g \sigma ; c_{1}, \ldots, c_{k}\right)=\lambda\left(g ; c_{\sigma^{-1}(1)}, \ldots, c_{\sigma^{-1}(k)}\right) \sigma\left\langle j_{1}, \ldots, j_{k}\right\rangle,
$$


where $\sigma\left\langle j_{1}, \ldots, j_{k}\right\rangle \in \Sigma_{j}$ is the permutation

$$
\mathbf{j} \cong \mathbf{j}_{1} \wedge \cdots \wedge \mathbf{j}_{k} \stackrel{\sigma}{\rightarrow} \mathbf{j}_{\sigma^{-1}(1)} \wedge \cdots \wedge \mathbf{j}_{\sigma^{-1}(k)} \cong \mathbf{j}
$$

the isomorphisms being lexicographic identifications.

(d) For each fixed $g \in \mathcal{G}_{k}$, the maps $\lambda(g)$ specify a $k$-fold product on $\mathcal{C}$, that is, a natural transformation $\varrho^{k} \rightarrow \circlearrowright \circ \wedge$, where $\wedge: \Lambda^{k} \rightarrow \Lambda$ is the $k$-fold smash product.

EXAMPLE 2.2. When $e$ is also an operad, only the analog of (a) involving the structural maps $\gamma$ of $\varrho$ needs to be added to make this definition coincide with the definition of an action of an operad $\mathcal{G}$ on an operad $\mathcal{C}([11, \mathrm{VI}, 1.6]$, with misprints corrected in $[13,1.8])$. Thus the operad pairs $(\mathcal{S}, \mathcal{G})$ used in [11] and [13] provide examples.

The following example is implicit in Lemma 1.8 .

Lemma 2.3. Let $\mathcal{C}$ be a weakly separated $\mathcal{G}$-module. Then $\mathcal{G}$ acts multiplicatively on the configuration space coefficient system $\mathrm{C}(\overline{\mathcal{C}})$.

Proof. The point $* \in \bar{\complement}_{0} \subset \mathcal{e}=F(\bar{\complement}, 1)$ is taken to be the unit of $\mathcal{C}(\bar{\complement})$. For $g \in \mathfrak{G}_{k}$, the maps $\lambda(g)$ are the "composites"

$$
F\left(\bar{\varrho}, j_{1}\right) \times \cdots \times F\left(\bar{\complement}, j_{k}\right) \stackrel{\otimes}{\rightarrow} F\left(\bar{\complement}^{k}, j\right) \stackrel{F(\gamma(g), j)}{\longrightarrow} F(\bar{\complement}, j)
$$

$j=j_{1} \cdots j_{k}$. The requisite verifications are straightforward.

Now the action of $\mathcal{G}$ on $C(\overline{\mathcal{C}}, X)$ of Lemma 1.8 is seen to be a special case of the following generic kind of action (which is a direct generalization of [11, VI.1.8]).

Lemma 2.4. Let $\mathcal{G}$ act multiplicatively on $\mathcal{C}$ and let $X$ be a $\mathfrak{G}_{0}$-space. Then $C X$ is functorially $a \mathcal{G}_{0}$-space and the natural map $\eta: X \rightarrow C X$ is a $\mathscr{S}_{0}$-map.

Proof. $C X$ is defined using the basepoint $0 \in X, \eta: X \rightarrow C X$ is specified by $\eta(x)=[1 ; x]$ and the zero and unit for $C X$ are the elements $\eta(0)=[* ; *]$ and $\eta(1)$. The action $\xi_{k}$ on $C X$ is specified in terms of $\lambda$ and the action $\xi_{k}$ on $X$ by the formula

$$
\xi_{k}\left(g ; \underset{r=1}{\chi}\left[c_{r} ; y_{r}\right]\right)=\left[\lambda\left(g ; \underset{r=1}{\chi} c_{r}\right) ; \underset{I}{\chi} \xi_{k}\left(g ; y_{I}\right)\right]
$$

for $g \in \mathcal{G}_{k}, c_{r} \in \mathcal{C}_{j_{r}}$, and $y_{r}=\left(x_{r, 1}, \ldots, x_{r, j_{r}}\right) \in X^{j_{r}}$, where $I$ runs over the lexicographically ordered set of sequences $\left(i_{1}, \ldots, i_{k}\right)$ with $1 \leqslant i_{r} \leqslant j_{r}$ and where $y_{I}=$ $\left(x_{1, i_{1}}, \ldots, x_{k, i_{k}}\right) \in X^{k}$. The requisite verifications are elementary exercises.

The following omnibus result, whose unexplained notations will be clarified in the course of the ensuing discussion, will be used in conjunction with Proposition 1.9.

Proposition 2.5. Let $\mathcal{C}$ be a weakly separated $\mathcal{G}$-module and $X$ a $\mathfrak{S}_{0}$-space. Then all of the following are $\mathcal{G} \times$-maps:

$$
C(\bar{\complement}, X) \stackrel{\pi_{1}}{\leftarrow} \tilde{C}(\bar{\complement}, X) \stackrel{\pi_{2}}{\rightarrow} K_{\infty} X \stackrel{\alpha_{\infty}}{\rightarrow} Q X
$$

Here $\mathcal{L}$ is the linear isometries operad of $\left[11\right.$, p. 10], so that $\mathcal{L}_{j}$ is the space of linear isometries $\left(R^{\infty}\right)^{j} \rightarrow R^{\infty}$. It is an $E_{\infty}$ operad; that is, $\varrho_{j}$ is $\Sigma_{j}$ free and contractible. In multiplicative infinite loop space theory, the little cubes operad $e_{\infty}$ used in [2] is an 
inappropriate choice for the canonical additive $E_{\infty}$ operad. The little convex bodies operad of [11] was a clumsy first approximation to the right choice. The definitive choice was introduced by Steiner [14]. It is denoted $\mathcal{K}_{\infty}$, and $K_{\infty} X$ denotes the associated construction on spaces. As in the proof of $[2,4.6], \tilde{C}(\bigodot, X)$ is constructed from the product coefficient system $\tilde{e}(\bar{\varrho})=e(\bar{\complement}) \times \mathscr{K}_{\infty}, \pi_{1}$ and $\pi_{2}$ are induced from the projections, and $\pi_{1}$ is a homotopy equivalence.

Of course, $\mathcal{G} \times \mathcal{L}$ acts multiplicatively on $\mathcal{C}(\overline{\mathcal{C}})$ by pullback along the projection $\mathcal{G} \times \mathcal{L} \rightarrow \mathcal{G}$. Similarly, $\mathcal{G} \times \mathcal{L}$ acts on the space $X$. It is a basic property of $\mathcal{K}_{\infty}$ that $\mathcal{L}$, and therefore also $\mathcal{G} \times \mathcal{E}$, acts on it. We conclude that $\mathcal{G} \times \mathcal{E}$ acts on $\tilde{\mathcal{C}}(\overline{\mathcal{C}})$ in such a way that the projections to $\mathcal{C}(\bar{C})$ and to $\mathscr{K}_{\infty}$ commute with the actions. Therefore $\pi_{1}$ and $\pi_{2}$ are maps of $\mathcal{G} \times \mathcal{L}$-spaces. It is another basic property of $\mathcal{K}_{\infty}$ that there is a natural map $\alpha_{\infty}: K_{\infty} X \rightarrow Q X$ which is a homotopy equivalence if $X$ is connected and a group completion in general. Moreover, $Q X$ is a $\mathcal{G} \times \mathcal{L}$-space and $\alpha_{\infty}$ is a $\mathcal{G} \times \mathcal{L}$-map when $X$ is a $\mathcal{G}_{0}$-space. These facts imply the proposition.

Actually, much more is true; $\left(\mathcal{K}_{\infty}, \mathcal{G} \times \mathfrak{L}\right)$ is an operad pair and $K_{\infty} X$ is the free $\left(K_{\infty}, \mathcal{G} \times \mathcal{L}\right)$-space generated by the $\mathcal{S}_{0}$-space $X ; \mathcal{Q} X$ is also a $\left(\mathcal{K}_{\infty}, \mathcal{G} \times \mathcal{L}\right)$-space and $\alpha_{\infty}: K_{\infty} X \rightarrow Q X$ is a $\left(K_{\infty}, \mathcal{G} \times \mathscr{E}\right)$-map. Intuitively, these statements mean that $K_{\infty} X$ and $Q X$ are $H$-semiring spaces with additions and multiplications parametrized by $\mathcal{K}_{\infty}$ and $\mathcal{G} \times \mathfrak{E}$. The parameter spaces encode higher homotopies, and $\alpha_{\infty}$ preserves all of this information. When $\mathcal{G}=\mathcal{C}_{n}$, a $\left(\mathscr{K}_{\infty}, \mathcal{G} \times \mathcal{L}\right)$-space is said to be an $E_{n}$ ring space. When $\mathcal{G}$ is an $E_{\infty}$ operad, a $\left(\mathscr{K}_{\infty}, \mathcal{G} \times \mathcal{L}\right)$-space is said to be an $E_{\infty}$ ring space. The interrelationship between the additive and multiplicative operad actions provides a very powerful tool for the analysis of the latter. We refer to [11-14] for details on all of this and turn to a discussion of its calculational significance in our context of James maps.

3. Implications, interpretations, and delooping theorems. Let $\mathcal{G}$ be an operad, $\mathcal{C}$ weakly separated $\mathcal{S}$-module, and $X$ a space with basepoint $*$. Consider the following commutative diagram:

(A)

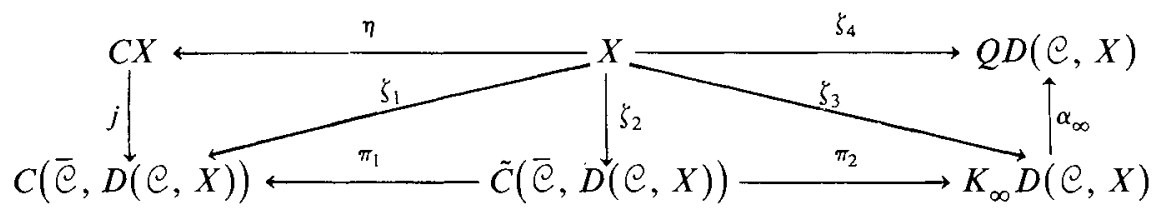

Here $\eta$ is the based inclusion, $\eta(x)=[1 ; x]$, determined by a unit $1 \in \bigodot_{1}$. Clearly $\eta$ induces a based inclusion, still denoted $\eta$, of $X$ in $D_{1}(\bigodot, X)$. If $\bigodot_{1}$ is contractible, as always holds in practice, the latter inclusion is a homotopy equivalence. Consider the composites

$$
X \stackrel{\eta}{\rightarrow} C X \stackrel{j_{q}}{\rightarrow} C\left(\overline{\mathrm{e}}_{q}, D_{q}(\bigodot, X)\right) .
$$

For $q=0, e_{0}=\{*\}$ and $j_{0}$ collapses all of $C X$ to the point $[\langle *\rangle, 1]$, where $1 \in S^{0}=D_{0}(\circlearrowright, X)$; compare $[2, \S 3]$. For $q=1$, direct calculation from the definitions gives

$$
\left(j_{1} \eta\right)(x)=[\langle 1\rangle, \eta(x)]
$$


Similarly, for $q>1, j_{q} \eta$ is constant at the basepoint. Thus we must define

$$
\zeta_{1}(x)=(j \eta)(x)=\left[\oplus \circ\left(j_{q}\right)\right](x)=[\langle *, 1\rangle ; 1, \eta(x)] .
$$

If $x=*$, this is the same point as the unit $[\langle *\rangle ; 1]$ of the $\mathcal{G}$-space $C(\overline{\mathcal{C}}, D(\mathcal{C}, X))$. To define $\zeta_{2}$ and $\zeta_{3}$, simply replace $\langle *, 1\rangle \in F(\overline{\mathcal{C}}, 2)$ on the right by $\left(\langle *, 1\rangle, k_{2}\right)$ and $k_{2}$, where $k_{2}$ is any chosen point of $K_{\infty, 2}$. Of course, $\zeta_{4}=\alpha_{\infty} \zeta_{3}$. Note that $\zeta_{i}(*)$ is connected to the unit by a path, but is not equal to the unit, if $i>1$. Indeed, as discussed in $\left[9\right.$, p. 4], a path in $\mathscr{K}_{\infty, 1}$ connecting $\gamma\left(k_{2} ; *, 1\right)$ to the unit 1 determines all of the required paths. If, as we assume, $*$ is a nondegenerate basepoint of $X$, we can replace $\eta$ and the $\zeta_{i}$ by homotopic maps such that the diagram is still strictly commutative but the new maps all carry $*$ to the unit of their $\operatorname{target} \mathcal{G} \times \mathcal{E}$-spaces. To see this, let $X^{\prime}=X \vee I$ with new basepoint at the end of the whisker $I$. Extend $\eta$ and $\zeta_{1}$ over the whisker by use of the constant path and extend $\zeta_{i}, i>1$, by use of the paths just indicated. There results a commutative based diagram with domain $X^{\prime}$. The projection $X^{\prime} \rightarrow X$ is a based homotopy equivalence, and precomposition with a chosen homotopy inverse gives the required new maps $\eta$ and $\zeta_{i}$.

Let $(G \times L)(X)$ denote the free $\mathcal{G} \times \mathcal{L}$-space generated by $X$. Since, by Propositions 1.9 and $2.5, j, \pi_{1}, \pi_{2}$, and $\alpha_{\infty}$ are all $\mathcal{G} \times \mathcal{L}$-maps, there results a commutative diagram of $\mathcal{G} \times \mathcal{L}$-spaces and $\mathcal{G} \times \mathcal{L}$-maps:

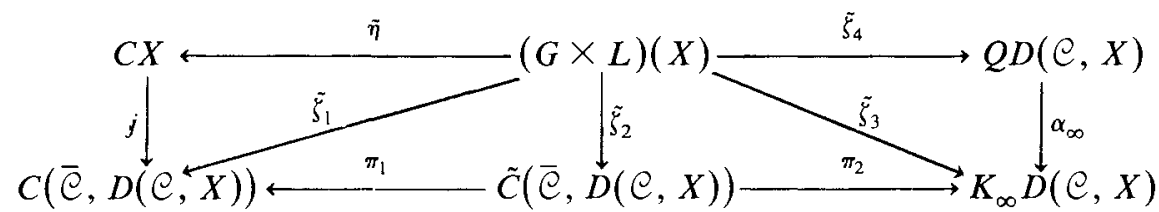

Choosing inverses to equivalences, let $j$ also denote the composite total James map $\alpha_{\infty} \pi_{2} \pi_{1}^{-1} j$. When $\mathcal{C}=\mathcal{G}$ is an operad, or when $\mathcal{C}=\mathcal{C}\left(R^{n}\right)$ and $\mathcal{G}=\mathcal{C}_{n}, \tilde{\eta}$ is an equivalence [4, 1.7]. In this case, $j$ is homotopic to $\tilde{\zeta}_{4} \eta^{-1}$. Here $\tilde{\zeta}_{4}$ is entirely determined by its restriction to $X$, which is completely understood, and by the $\mathcal{G} \times \mathcal{E}$-structure on the $\left(\mathscr{K}_{\infty}, \mathcal{G} \times \mathcal{L}\right)$-space $Q D(\mathcal{C}, X)$.

To see the calculational significance of this assertion, consider $\bmod p$ homology for a prime $p$. If $\mathcal{C}$ is an $E_{\infty}$ operad, then $H_{*} C X$ is a functor of $H_{*} X$. More precisely, $H_{*} C X$ is functorially generated by $\tilde{H}_{*} X$ via the Pontryagin product and homology operations determined by the action of $\mathcal{C}$ on $C X$. See [3, I.4.1]. We think of this structure as additive. With $\mathcal{C}=\mathcal{G}$, we think of the resulting $\mathcal{G}$-action on $D(\mathcal{C}, X)$ of Lemma 1.7 as multiplicative. The induced multiplicative Pontryagin product and homology operations on $H_{*} D(\mathcal{C}, X)$ can be read off by passage to filtration quotients from the additive structure on $H_{*} C X$. Precisely analogous assertions, but with a more complicated set of homology operations involved, are valid when $\mathrm{e}=\mathrm{e}_{n}=\mathcal{G}$. See [3, III.3.1].

Now consider $Q X$. Again $H_{*} Q X$ is functorially generated by $\tilde{H}_{*} X$ via the (additive) Pontryagin product and homology operations determined by the action of $\mathscr{K}_{\infty}$. See [3, I.4.2]. For any $E_{\infty}$ ring space, the general formulas of [3, II, §§1-3] determine the multiplicative Pontryagin product and homology operations on elements decomposable under the additive Pontryagin product and homology operations. Although the details have yet to be worked out, it is clear from the geometric 
diagrams on hand that such formulas must also exist for $E_{n}$ ring spaces. When $X$ is a $\mathcal{G}_{0}$-space, $Q X$ is a $\left(\mathscr{K}_{\infty}, \mathcal{G} \times \mathfrak{L}\right)$-space; when $\mathcal{G}$ is $\mathcal{C}_{n}$ or an $E_{\infty}$ operad, we conclude that the multiplicative Pontryagin product and homology operations on $H_{*} Q X$ are algorithmically determined from this structure on $H_{*} X$. See [3, II, §4] for a more detailed discussion.

Returning to $Q D(\mathcal{C}, X)$, we conclude that if $\mathcal{C}=\mathcal{G}$ is $\mathcal{C}_{n}$ or an $E_{\infty}$ operad, then the multiplicative Pontryagin product and homology operations on $H_{*} Q D(\bigodot, X)$ are algorithmically determined by $H_{*} X$. The total James map $j: C X \rightarrow Q D(\bigodot, X)$ converts the additive structure on $H_{*} C X$ to the multiplicative structure on $H_{*} Q D(\mathcal{e}, X)$, and we know the restriction of $j_{*}$ to $H_{*} X$. We therefore have an algorithm for the global computation of $j_{*}$, To compute the $q$ th James map on homology, we first compute the total map and then project to the $q$ th coordinate via $D(\mathcal{C}, X) \rightarrow D_{q}(\mathcal{C}, X)$.

To see what is going on more clearly, it is useful to redescribe $H_{*} Q D(\mathcal{C}, X)$ in terms of the composite equivalence

$$
\prod_{q \geqslant 0} Q D_{q}(\mathcal{e}, X) \stackrel{\Pi_{q} Q i_{q}}{\rightarrow} \prod_{q \geqslant 0} Q D(\mathcal{C}, X) \stackrel{*}{\rightarrow} Q D(\mathcal{C}, X),
$$

where $i_{q}: D_{q}(e, X) \rightarrow D(e, X)$ is the inclusion and $*$ is the loop sum. Up to homotopy, the map $\zeta_{4}$ of diagram (A) is the composite of this equivalence and the map $X \rightarrow \Pi_{q \geqslant 0} Q D_{q}(\mathcal{C}, X)$ with coordinates $(1, \eta, 0, \ldots, 0, \ldots)$, where 1 denotes the constant map at $1 \in S^{0} \subset Q S^{0}, \eta$ denotes the composite $X \stackrel{\eta}{\rightarrow} D_{1}(\bigodot, X) \subset$ $Q D_{1}(\mathrm{e}, X)$, and the remaining coordinates are constant at 0 . Since we are using weak infinite products,

$$
H_{*}\left(\prod_{q \geqslant 0} Q D_{q}(\circlearrowright, X)\right)=\bigotimes_{q \geqslant 0} H_{*} Q D_{q}(e, X) .
$$

In $H_{*} Q D(\mathcal{C}, X)$, the tensor products here become loop sums, in line with the fact that $H_{*} Q D(\bigodot, X)$ is generated under the additive Pontryagin product and homology operations by

$$
\tilde{H}_{*} D(\mathcal{C}, X)=\sum_{q \geqslant 0} \tilde{H}_{*} D_{q}(\bigodot, X)
$$

We refer to Kuhn $[6,7]$ for concrete calculations and geometric applications based on these ideas and turn to homotopical, rather than homological, implications of our study of operad actions.

If $e_{n} \times \mathcal{C}$ acts on a space $X$, then there is an $n$-fold classifying space $B^{n} X=$ $B\left(\Sigma^{n}, C_{n} \times L, X\right)$ and a natural map $\iota: X \rightarrow \Omega^{n} B^{n} X$ which is a weak equivalence if $X$ is connected and a group completion in general (if $n \geqslant 2$ ). See [9, §13] and, for the nonconnected case, $[10, \S 2]$ and $[3$, p. 487, \#11]. In our theory we often begin with multiplicative actions by $e_{n} \times \varrho$ on ring spaces $X$. Unless we shrink to appropriate unit components, $\Omega^{n} B^{n} X$ will be trivial (due to the presence of zero).

Identify $\Pi_{q \geqslant 1} Q D_{q}(\mathcal{C}, X)$ with the unit subspace of $\Pi_{q \geqslant 0} Q D_{q}(\mathcal{C}, X)$, namely the subspace consisting of those points whose 0 th coordinate is 1 . A compatible embedding of $Q\left(\vee_{q \geqslant 1} D_{q}(e, X)\right)$ in $Q D(\mathcal{C}, X)$ is obtained by mapping a point $f$ to 
the loop sum $1 *(Q i)(f)$, where $i: \vee_{q>1} D_{q}(\mathcal{C}, X) \rightarrow D(\mathcal{C}, X)$ is the natural based inclusion and 1 is the unit element. We write

$$
U Q D(e, X) \simeq Q\left(\underset{q \geqslant 1}{\vee} D_{q}(e, X)\right)
$$

for this unit space.

Upon restriction to unit subspaces of the targets of the maps $\tilde{\zeta}_{i}$, we can apply the functor $B^{n}$ to the entire diagram (B) when $\mathcal{G}=e_{n}$. In particular, inverting equivalences, we obtain a map $B^{n} j: B^{n} C X \rightarrow B^{n} U Q D(\mathcal{C}, X)$ and a homotopy commutative diagram

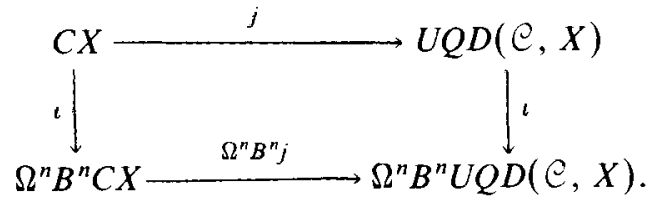

Thus $j$ becomes an $n$-fold loop map upon group completion. If $X$ is connected, the maps $\iota$ are equivalences and we conclude that $j$ itself is an $n$-fold loop map.

When $\mathcal{G}=\bigodot_{\infty}$, or any other $E_{\infty}$ operad, we can carry out precisely the same discussion with $n=\infty$. Here $B^{\infty}$ is to be interpreted as the spectrum-valued functor of $[9, \S 14]$, and $\Omega^{\infty}$ is to be interpreted as the 0th space functor from spectra to spaces. In this case, $j$ is an infinite loop map if $X$ is connected.

At this writing, these facts are more curious than useful, and further investigation is certainly called for. One would like to identify the spaces involved, in particular $B^{n} C X$ and $\Omega^{n} B^{n} U Q D(\mathcal{C}, X)$. For the latter, we have the following weakly homotopy commutative diagram (at least if $\pi_{0} X$ is countable):

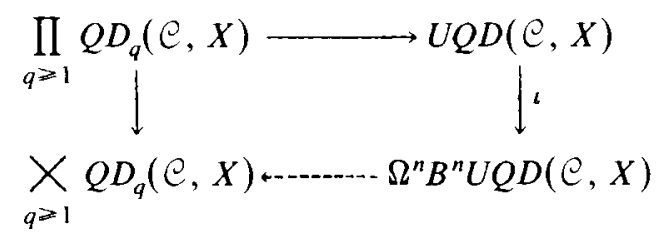

Here the left arrow is the inclusion of the weak infinite product in the full infinite product. We proved in $[2,1.7]$ that the latter is a grouplike multiplicative $H$-space, and $[2,1.2]$ gives the dotted arrow. It seems reasonable to conjecture that the dotted arrow is a weak equivalence. If $X$ is connected, then $D_{q}(\mathcal{e}, X)$ is $(q-1)$-connected and all maps in the diagram are weak equivalences. In general, verification of the conjecture would require better understanding of the homology of infinite products than we possess.

We can identify $B^{n} C X$ in many interesting cases. If $\varrho=e_{n}$, then $B^{n} C X$ is equivalent to $\Sigma^{n} X$ by $[9,13.1$ (vii)]. Of course, this conclusion carries over to the case $e=e\left(R^{n}\right)$. Much more generally, Caruso [1] has proven that $B^{n} C\left(R^{n} \times Y, X\right)$ is equivalent to $C\left(Y, \Sigma^{n} X\right)$ for any weak metric space $Y$. 


\section{BIBLIOGRAPHY}

1. J. Caruso, Configuration spaces and mapping spaces, Thesis, Univ. of Chicago, 1979.

2. J. Caruso, F. R. Cohen, J. P. May and L. R. Taylor, James maps, Segal maps, and the Kahn-Priddy theorem, Trans. Amer. Math. Soc. 281(1984), 243-283.

3. F. R. Cohen, T. Lada and J. P. May, The homology of iterated loop spaces, Lectures Notes in Math, vol. 533, Springer-Verlag, Berlin and New York, 1976.

4. F. R. Cohen, J. P. May and L. R. Taylor, Splitting of certain spaces $C X$, Math. Proc. Cambridge Philos. Soc. 84 (1978), 465-496.

5. R. L. Cohen, Stable proofs of stable splittings, Math. Proc. Cambridge Philos. Soc. 88 (1980), 149-152.

6. N. J. Kuhn, The homology of the James-Hopf maps, Illinois J. Math. 27 (1983), 315-333.

7.,$A$ Kahn-Priddy sequence and a conjecture of $G$. W. Whitehead, Math. Proc. Cambridge Philos. Soc. 92 (1982), 467-483.

8. L. G. Lewis, Jr., J. P. May, J. McClure and M. Steinberger, Equivariant stable homotopy theory, Lecture Notes in Math. (to appear).

9. J. P. May, The geometry of iterated loop spaces, Lecture Notes in Math., vol. 271, Springer-Verlag, Berlin and New York, 1972.

10. ____ $E_{\infty}$ spaces, group completions and permutative categories, London Math. Soc. Lecture Notes Ser., no. 11, Cambridge Univ. Press, Cambridge, 1974, pp. 61-93.

11. ___ (with contributions by F. Quinn, N. Ray and J. Tornehave), $E_{\infty}$ ring spaces and $E_{\infty}$ ring spectra, Lecture Notes in Math., vol. 577, Springer-Verlag, Berlin and New York, 1977.

12. ___ Pairings of categories and spectra, J. Pure Appl. Algebra 19 (1980), 199-346.

13.

14. R. Steiner, A canonical operad pair, Math. Proc. Cambridge Philos. Soc. 86 (1979), 443-449.

Department of Mathematics, University of Kentucky, LeXington, Kentucky 40506

Department of Mathematics, University of Chicago, Chicago, Illinois 60637

Department of Mathematics, University of Notre Dame, Notre Dame, Indiana 46556 